\title{
Dynamical effective parameters of elastic superlattice with strong acoustic contrast between the constituents
}

\author{
Yurii Zubov $^{1}$, Bahram Djafari-Rouhani ${ }^{2}$, and Arkadii Krokhin ${ }^{1}$ \\ ${ }^{1}$ Department of Physics, University of North Texas, P.O. Box 311427, Denton, TX 76203 \\ ${ }^{2}$ Université de Lille, Department of Physics, Villeneuve d'Ascq 59655, France \\ E-mail: arkady@unt.edu
}

Received July 9, 2018, published online October 26, 2018

\begin{abstract}
Analytical formulas are obtained for frequency-dependent effective elastic modulus and effective mass density for a periodic layered structure. The proposed homogenization procedure is valid at sufficiently high frequencies well above the lowest band gap in the acoustic spectrum of the structure. It is shown that frequencydependent effective parameters may take negative values either in different regions of frequencies or in the same quite narrow region. This property demonstrates that $1 \mathrm{D}$ elastic structure may behave in the limit of small Bloch wave vectors as a double-negative acoustic metamaterial.
\end{abstract}

Keywords: elastic superlattice, elastic modulus, periodic layered structure.

\section{Introduction}

Elastic periodic structures or phononic crystals originally were considered as passive environment where sound waves of certain region of frequencies cannot propagate because of presence of prohibited zones (band gaps) in their spectra of vibrations [1-3]. Now a variety of periodic elastic structures serve as principal elements of different acoustic devices operating in the regions of frequencies where sound waves can propagate and where they exhibits "exotic" properties, like anomalous dispersion, negative refraction, strong anisotropy, etc. If these properties can be explained within the effective medium theory, it is worthwhile to calculate the effective parameters of this homogeneous medium. For longitudinal waves it is sufficient to know the effective mass density $\rho_{\text {eff }}(\omega)$ and the effective elastic modulus $\lambda_{\text {eff }}(\omega)$, which are the functions of frequency $\omega$. For a long time a selfconsistent approach to homogenization of elastic composites proposed by Barryman for spherical [4] and ellipsoidal scatterers [5] was widely used. This long-wavelength limit is valid for the composites with low concentration of scatterers in space, when the details of the microstructure of the composite are ignored. This approach also does not lead to frequency dispersion, giving the result for $\rho_{\text {eff }}(0)$ and $\lambda_{\text {eff }}(0)$, i.e., the quasistatic limit for the effective parameters. Phononic band structure engineering and rapid development of new acoustic and elastic metamaterials gave rise to more advanced effective medium theories.

Homogenization theories based on plane or cylindrical wave expansions were developed in Refs. 6-11 for calcula-

(C) Yurii Zubov, Bahram Djafari-Rouhani, and Arkadii Krokhin, 2018 tions of the effective parameters of two- and threedimensional elastic periodic structures in the quasistatic limit. The effective parameters are obtained by taking the limit $\omega \rightarrow 0, k \rightarrow 0$ in the exact wave equation written for periodic structure, therefore they take into account all the details of distribution of elastic material within the corresponding unit cell.

The effective parameters obtained in the quasi-static limit are dispersionless and they are not valid for calculation of the bandgaps. The latter requires application of the dynamical methods. Frequency-dependent effective parameters for 2D elastic systems were calculated in Ref. 12. A two-scale approach proposed in this paper is valid for quite high frequencies $\omega$ and wave vector $\mathbf{k}$ lying near the edges of the Brillouin zone. More general plane-wave expansion approach valid in 3D case and for arbitrary $\omega$ and $\mathbf{k}$ was developed by Norris et al. [13]. An alternative approach for calculation of the effective parameters of phononic metasolids with local resonances was proposed in Ref. 14. It was demonstrated that these structures are described by means of frequency-dependent and anisotropic effective mass density, stifness tensor, and a third rank coupling tensor that is a signature of a nonlocal Willis medium.

So far, the approaches developed in Refs. 12-14 give the most complete description of a periodic structure by its effective parameters. The other side of wide generality of the reported results is that they require quite extensive numerical procedure. This is the reason why only one-dimensional structures were considered in Refs. 12,13 as examples of application of the proposed methods. Less general but more 
feasible methods of homogenization of 2D and 3D phononic crystals are proposed in Refs. 15,16 and Ref. 17, respectively. Unlike the exact results [13] these methods are based on different approximations. Nevertheless, they reproduce with high accuracy the band structures of 2D and 3D phononic crystals in the long-wavelength limit.

Layered (one-dimensional) periodic structures are of special interest as they are significantly easier and less expensive to fabricate than 2D or 3D structures. Theoretical study of acoustic properties of layered structures is also simplified due to the well-known dispersion relation $\omega=\omega(k)$. For a binary composite it has the following form [18]:

$$
\cos (k d)=\cos \left(\frac{\omega a}{c_{a}}\right) \cos \left(\frac{\omega b}{c_{b}}\right)-\frac{Z_{a}^{2}+Z_{b}^{2}}{2 Z_{a} Z_{b}} \sin \left(\frac{\omega a}{c_{a}}\right) \sin \left(\frac{\omega b}{c_{b}}\right) .
$$

Here $d=a+b$ is the period of the structure containing two layers of width $a$ and $b, c_{a}\left(c_{b}\right)$ is the speed of sound in the layer $a(b)$, and $Z_{a}=\rho_{a} c_{a}\left(Z_{b}=\rho_{b} c_{b}\right)$ is the acoustic impedance of the layer $a(b)$. In an infinite periodic structure Eq. (1) defines the dispersion of a Bloch wave of pressure propagating perpendicular to the layers, $p(x, t)=$ $=u_{k}(x) \exp [i(k x-\omega t]$.

In the quasi-static limit the effective density and elastic modulus of layered structure are easily calculated from Eq. (1) by taking the limit $\omega, k \rightarrow 0$ and assuming that $\omega / k=c_{\text {eff }}=$ const,

$$
\rho_{\text {eff }}=\frac{a}{d} \rho_{a}+\frac{b}{d} \rho_{b}, \quad \frac{1}{\lambda_{\text {eff }}}=\frac{a / d}{\lambda_{a}}+\frac{b / d}{\lambda_{b}} .
$$

These formulas are valid only for the lowest (acoustic) band, where dispersion of sound is linear, $\omega=c_{\mathrm{eff}} k$, $c_{\text {eff }}=\sqrt{\lambda_{\text {eff }} / \rho_{\text {eff }}}$.

For higher (optical) bands, the effective parameters exhibit dispersion, i.e., $\rho_{\text {eff }}=\rho_{\text {eff }}(\omega), \lambda_{\text {eff }}=\lambda_{\text {eff }}(\omega)$. Frequency-dependent effective parameters of $1 \mathrm{D}$ phononic crystals have been studied in many publications $[12,13,16,19,20]$ where the results for $\rho_{\text {eff }}(\omega)$ and $\lambda_{\text {eff }}(\omega)$ are presented in a graphical form. Analytical results for the effective parameters beyond the quasi-static limit (2) are still lacking, while for $1 \mathrm{D}$ case the dispersion Eq. (1) is known explicitly.

Here we propose a method of homogenization which for periodic layered medium leads to simple analytical formulas for the effective parameters. We introduce the effective medium through two principal equations of elastodynamics: equation of motion and Hooke's law. Propagating Bloch wave of pressure $p(x, t)$ induces oscillations of every element of the unit cell. Spatial period, phase and amplitude of these oscillations vary over the unit cell according to distribution of elastic material. Then, the acceleration $a_{u . c}$ and deformation $\Delta V / V$ of any local infinitesimally small layer within the unit cell are also functions of $x$. Replacing a periodic medium by a homogeneous one we request that in the long-wavelength limit $k d \ll 1$ both media exhibit the same dynamics. This requirement means that i) the average acceleration $\bar{a}$ equals to average acceleration of the effective medium subject to propagating plane (not Bloch) wave with the same wave vector $k$ and ii) the average deformation $\bar{\Delta} V / V$ equals to that of the effective medium. These two requirements provide that the unit cell as a whole moves as the corresponding part of the homogeneous effective medium. Two effective parameters, $\rho_{\text {eff }}(\omega)$ and $\lambda_{\text {eff }}(\omega)$, are obtained as a result of the above-mentioned requirements. Similar homogenization scheme was proposed in Ref. 21 for periodic arrangement of solid cylindrical scatterers.

For a wide region of frequencies the proposed effective parameters lead to a nonlinear dispersion relation

$$
\omega=k c_{\text {eff }}(\omega), \quad c_{\text {eff }}(\omega)=\sqrt{\lambda_{\text {eff }}(\omega) / \rho_{\text {eff }}(\omega)},
$$

which reproduces well the exact dispersion relation $\omega=\omega(k)$ obtained from Eq. (1), if $k d \ll 1$. This correspondence is valid not only within the passing bands but also within the band gaps where the wave vector $\mathbf{k}$ is pure imaginary since one of the effective parameters becomes negative. For a given combination of elastic materials in the unit cell the proposed formulas predict the region of frequencies where both effective parameters are negative, i.e., the layered structure behaves as metamaterial with negative index of refraction. So far the explicit frequency dependencies of the effective parameters were reported for acoustic devices exhibiting negative refraction near the frequencies of the internal resonances [22-24]. Reported here results is one more example of a periodic structure where the dispersion of the effective parameters can be calculated analytically.

\section{High-frequency homogenization scheme}

We consider a binary periodic composite of two elastic materials, $a$ and $b$ shown in Fig. 1 . The distribution of materials within the unit cell always can be selected symmetrical since the crystal as a whole possesses inverse symmetry. Propagating Bloch wave produces inhomogeneous distribution of pressure within the unit cell. Labeling the layers in the unit cell by index $n(n=1,2,3)$, the pressure can be written as follows:

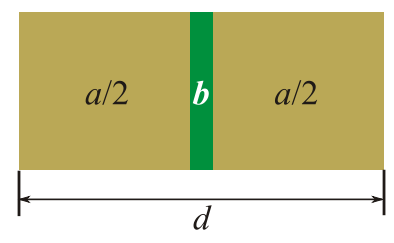

Material $a$, water: $\lambda_{a}=2 \cdot 10^{9} \mathrm{~Pa}$ $\rho_{a}=1000 \mathrm{~kg} / \mathrm{m}^{3}$ $a=10 \mathrm{~mm}$

- Material $b$, steel: $\lambda_{b}=2 \cdot 10^{11} \mathrm{~Pa}$ $\rho_{b}=7900 \mathrm{~kg} / \mathrm{m}^{3}$ $b=1 \mathrm{~mm}$

Fig. 1. (Color online) Symmetric unit cell of a binary composite. 


$$
p_{n}(x, t)=p_{n}(x) \mathrm{e}^{-i \omega t}, \quad p_{n}(x)=A_{n} \mathrm{e}^{i k_{n} x}+B_{n} \mathrm{e}^{-i k_{n} x},
$$

where the wave vector $k_{n}=\omega / c_{n}$ and $c_{n}=\sqrt{\lambda_{n} / \rho_{n}}$ is speed of sound in the $n$th layer. A set of linear equations for six constants $A_{n}$ and $B_{n}$ is obtained from the continuity of velocity and pressure at the layer interfaces, $x= \pm b / 2$

$$
\begin{gathered}
\left.\frac{1}{i \omega \rho_{n}} \frac{d p_{n}(x)}{d x}\right|_{x= \pm b / 2}=\left.\frac{1}{i \omega \rho_{n^{\prime}}} \frac{d p_{n^{\prime}}(x)}{d x}\right|_{x= \pm b / 2}, \\
p_{n}(x= \pm b / 2)=p_{n^{\prime}}(x= \pm b / 2),
\end{gathered}
$$

and the quasiperiodicity condition (Bloch theorem), $p_{n}(x+d)=\mathrm{e}^{i k d} p_{n}(x)$, at $x=-(a+b) / 2$. Since the equations in this set are homogeneous a nontrivial solution exists if the determinant vanishes, that leads to the dispersion Eq. (1). Once $A_{n}$ and $B_{n}$ are calculated, the pressure $p_{n}(x)$ in each layer can be expressed through, e,g., pressure at the left edge of the unit cell, $p_{1}\left(-\frac{a+b}{2}\right)=A_{1} \mathrm{e}^{i k_{a}(a+b) / 2}+$ $+B_{1} \mathrm{e}^{-i k_{a}(a+b) / 2}$.

In the long-wavelength limit, $k d \ll 1$ the Bloch wave in a periodic structure is weakly modulated and can be approximated by a plane wave that propagates in the equivalent effective medium. We propose to obtain the density of the effective medium by equating the average acceleration of the unit cell to that of the effective homogeneous layer of width $d$, assuming that the amplitude of the plane wave coming from the left is $-p_{1}\left(\frac{a+b}{2}\right)$. The local acceleration of elastic medium at point $x$ is $-\frac{1}{\rho(x)} \frac{d p(x)}{d x}$. Then, the average acceleration of the unit cell is obtained by integration

$$
\begin{gathered}
\bar{a}_{\text {u.c. }}=-\frac{1}{d} \int_{-\frac{a+b}{2}}^{\frac{a+b}{2}} \frac{d p_{n}(x)}{d x} \frac{1}{\rho_{n}(x)} d x= \\
=-\frac{1}{\rho_{a} d}\left(\mathrm{e}^{i k d}-1\right) p_{1}\left(-\frac{a+b}{2}\right)-\frac{1}{d}\left(\frac{1}{\rho_{b}}-\frac{1}{\rho_{a}}\right)\left[p_{2}\left(\frac{b}{2}\right)-p_{2}\left(-\frac{b}{2}\right)\right],
\end{gathered}
$$

Average acceleration of a homogeneous layer with density $\rho_{\text {eff }}$ in the field of the plane wave $p(x)=p_{1}\left(-\frac{a+b}{2}\right) \exp \left[i k\left(x+\frac{a+b}{2}\right)\right]$ is calculated in a similar way

$$
\begin{aligned}
& \bar{a}_{\text {eff }}=-\frac{1}{d \rho_{\mathrm{eff}}} \int_{-\frac{a+b}{2}}^{\frac{a+b}{2}} \frac{d p(x)}{d x} d x= \\
& =-\frac{1}{d \rho_{\mathrm{eff}}} p_{1}\left(-\frac{a+b}{2}\right)\left(\mathrm{e}^{i k d}-1\right) .
\end{aligned}
$$

The pressure $p_{1}\left(-\frac{a+b}{2}\right)$ can be selected as a constant through which all the unknowns $A_{n}$ and $B_{n}$ can be ex- pressed. Then the distribution of pressure within the unit cell is proportional to $p_{1}\left(-\frac{a+b}{2}\right)$ and this common factor is canceled when equating the accelerations given by Eqs. (6) and (7). Thus, the condition $\bar{a}_{\text {u.c. }}=\bar{a}_{\text {eff }}$ defines the effective density $\rho_{\text {eff }}(\omega)$.

One more mechanical characteristic of elastic medium is the field of deformation. For a layered inhomogeneous system the relative change of volume of the unit cell is obtained from the Hooke's law:

$$
\left(\frac{\overline{\Delta V}}{V}\right)_{\text {u.c. }}=\frac{1}{d} \int_{-\frac{a+b}{2}}^{\frac{a+b}{2}} \frac{p_{n}(x)}{\lambda(x)} d x,
$$

The same quantity for a homogeneous medium is given by

$$
\left(\frac{\overline{\Delta V}}{V}\right)_{\mathrm{eff}}=\frac{1}{d \lambda_{\mathrm{eff}}} \int_{-\frac{a+b}{2}}^{\frac{a+b}{2}} p(x) d x .
$$

The equation $\left(\frac{\overline{\Delta V}}{V}\right)_{\text {u.c. }}=\left(\frac{\overline{\Delta V}}{V}\right)_{\text {eff }}$ defines the effective bulk modulus $\lambda_{\text {eff }}(\omega)$. Thus, the proposed homogenization scheme is based on Eqs. (6)-(9) which establish equivalency in the dynamics of the unit cell and effective medium in the field of propagating sound wave. Apparently, that this method of homogenization is valid in the long-wavelength limit $k d \ll 1$ and within a wide range of frequencies (not necessarily low). The latter means that the elastic field inside each material layer can be strongly inhomogeneous, i.e., the parameters $k_{a} a=\omega a / c_{a}$ and $k_{b} b=\omega b / c_{b}$ are not necessarily small. It is clear that the proposed method is valid not only for layered structures; it is equally applicable for 2D [21] and 3D phononic crystals. In 1D case all the calculations can be done analytically and explicit expressions for the effective parameters are obtained.

\section{The effective parameters}

Solution of a homogeneous $6 \times 6$ set of linear equations for the coefficients $A_{n}$ and $B_{n}$ is a trivial but combersome problem. Omitting simple algebra, we give the final results for the dynamic effective parameters for the most interesting situation of high acoustic contrast between the constituents, $c_{a} \ll c_{b}$,

$$
\begin{gathered}
\rho_{\mathrm{eff}}(\omega)=\frac{\rho_{a} a+\rho_{b} b \frac{\omega a}{2 c_{a}} \cot \left(\frac{\omega a}{2 c_{a}}\right)}{a+b \frac{\left(\omega a / 2 c_{a}\right)}{\sin \left(\omega a / 2 c_{a}\right)}\left(1-\frac{2 \rho_{b}}{\rho_{a}} \sin ^{2}\left(\frac{\omega a}{4 c_{a}}\right)\right)}, \\
\lambda_{\text {eff }}^{-1}(\omega)=\frac{b \lambda_{b}^{-1}+a \lambda_{a}^{-1}\left(\frac{2 c_{a}}{\omega a}\right) \sin \left(\frac{\omega a}{2 c_{a}}\right)}{(a+b) \cos \left(\frac{\omega a}{2 c_{a}}\right)} .
\end{gathered}
$$


These formulas are valid in the long-wavelength limit $k d \ll 1$ for the structure with high acoustic contrast. The latter condition, $\omega b / c_{b} \ll 1$, means that that the layer with more stiff material (layer $b$ ) homogenizes, i.e. the acoustic field inside it is practically homogeneous. However, the field inside the softer layer can exhibit oscillatory pattern. This gives rise to frequency dispersion of the effective parameters. Of course, in the limit, $\omega, k \rightarrow 0$ Eqs. (10), (11) are reduced to the result of the quasi-static limit Eq. (2).

\section{Frequency dispersion of the effective parameters}

To study the dispersion of the effective parameters we select a structure containing layers of water (material $a$, thickness $a=10 \mathrm{~mm}$ ) and steel (material $b$, thickness $b=1 \mathrm{~mm})$. The acoustic contrast between these two materials is rather high: $c_{b} / c_{a} \approx 3.5$. The geometrical and physical parameters of the unit cell are shown in Fig. 1. Due to high contrast and different thicknesses there is a wide range of frequencies where the obtained formulas for the effective parameters are valid. The homogenization condition for the water layer $\omega a / c_{a}<1$ is true for frequencies $\omega_{\min } / 2 \pi<2.5 \cdot 10^{4} \mathrm{~Hz}$. At such low frequencies the results (2) obtained in the quasi-static limit are valid. Indeed, the effective parameters in Fig. 2 do not exhibit essential dispersion up to $2 \cdot 10^{4} \mathrm{~Hz}$. Here the dispersion of sound is linear, see Fig. 2(c). At higher frequencies both effective parameters exhibit strong frequency dispersion, which originates from inhomogeneity of acoustic field in water layers. Unlike this, the field within the steel layers is homogeneous, i.e., $\omega b / c_{b} \ll 1$. This condition is violated at frequencies higher than $\omega_{\max } / 2 \pi=c_{b} / 2 \pi b \approx 0.8 \mathrm{MHz}$. Thus, within the range from $20 \mathrm{kHz}$ to $0.8 \mathrm{MHz}$ the effective parameters are frequency-dependent and they can be calculated using Eqs. (10) and (11).

Of course, these results are valid only in the longwavelength limit, i.e., in the vicinity of the $\Gamma$-point. Therefore, the parts of the graphs lying in the region of frequencies corresponding to the edges of the Brillouin zone (where $k d$ close to $\pi$ ) should be ignored. Within the band gap (shaded region close to the $\Gamma$-point in the Fig. 2(c)) the effective elastic modulus is negative but the effective density is positive. This leads to pure imaginary phase velocity, $V_{\mathrm{ph}}=\sqrt{\lambda_{\text {eff }} / \rho_{\text {eff }}}$, therefore the system is opaque sound wave does not propagate. The effective elastic modulus remains negative even below the lower edge of the gap ( $\approx 96 \mathrm{kHz}$ ). The effective density vanishes exactly at this frequency, going to negative values below it. Thus, there is a narrow region of frequencies where both effective parameters are negative and the structure is transparent to sound. This narrow region (below $100 \mathrm{kHz}$ ) is light shaded in the left and central panels. One more very narrow region with double-negative parameters lies near $370 \mathrm{kHz}$. Note that within these regions the dispersion of sound is anomalous, i.e., the phase and group velocities have opposite directions. Negative values of $\rho_{\text {eff }}$ and $\lambda_{\text {eff }}$ give rise to neg-

(a)

(b)

(c)

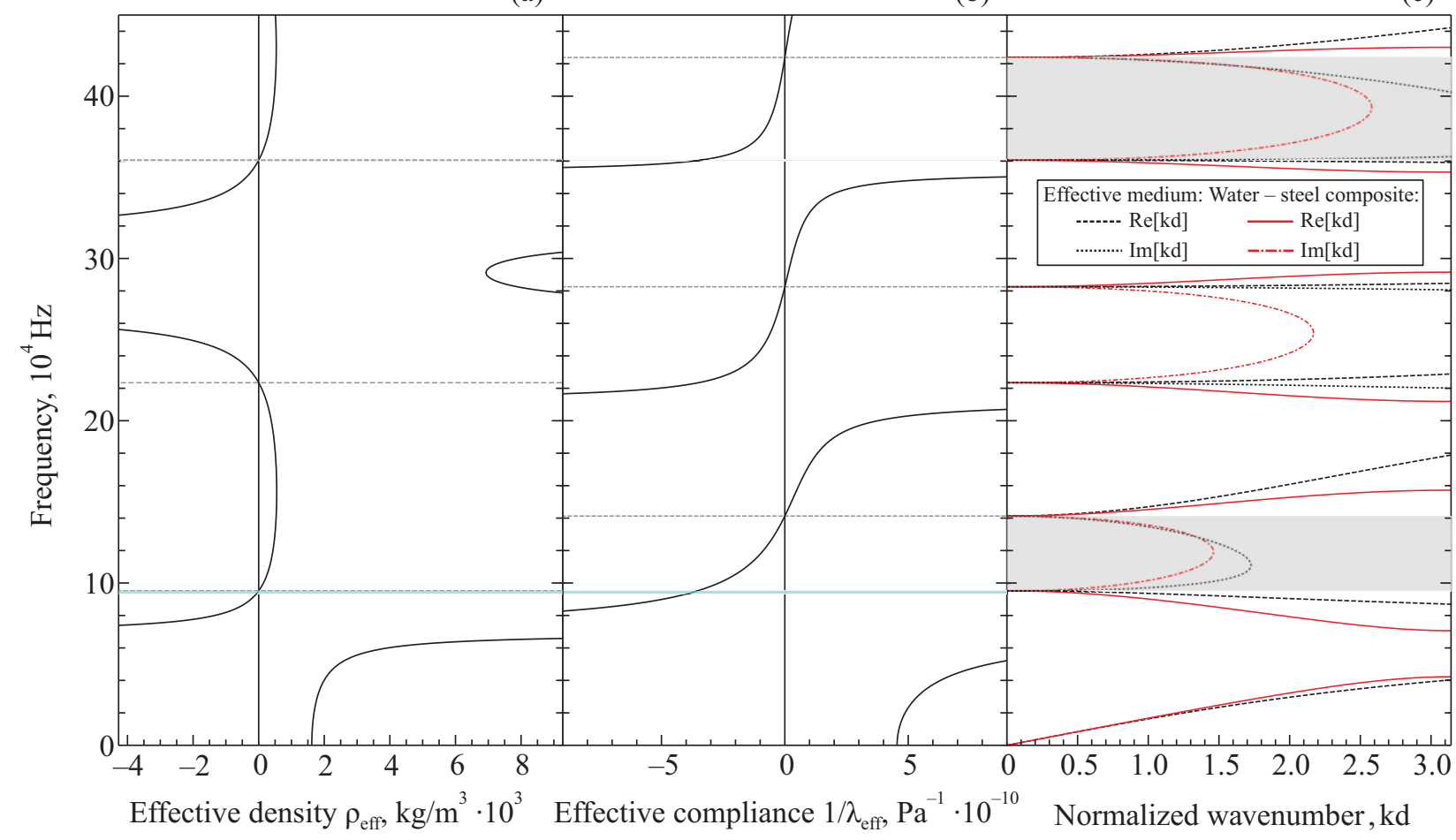

Fig. 2. (Color online) Effective density $\rho_{\text {eff }}(\omega)$ (a); effective compliance $\lambda_{\text {eff }}^{-1}(\omega)$ (b); dispersion curves for the water-steel periodic structure (solid lines) and for the effective medium (dashed lines) with parameters calculated using Eqs.(10) and (11) (c). Bandgap region is dark-shaded on (c). Two narrow regions (near 100 and $370 \mathrm{kHz}$ ) of double-negative effective parameters are light-shaded in (a) and (b) panels. 
ative refraction first predicted by Veselago for dielctrics with negative permittivity and permeability [25]. Negative refraction of sound was experimentally observed in 2D phononic crystals [26-28]. We are not aware of experimental observation on negative refraction in layered elastic structures. Double negative effective parameters for a layered structure were theoretically predicted in Ref. 20.

\section{Dispersion relation for sound wave in the long-wavelength limit}

Dispersion of sound propagating in a homogeneous (effective) medium is obtained from the relation:

$$
\frac{\omega}{k}=V_{\mathrm{ph}}=\sqrt{\frac{\lambda_{\mathrm{eff}}(\omega)}{\rho_{\mathrm{eff}}(\omega)}} .
$$

Plugging Eqs. (10) and (11) into Eq. (12) gives an explicit form of $k(\omega)$ function. The Bloch wave vector $k(\omega)$ is real only if $\rho_{\text {eff }}$ and $\lambda_{\text {eff }}$ are of the same sign.

Dispersion of sound obtained from Rytov equation (1) and from the effective-medium theory Eq. (12) is plotted in Fig. 2(c). Comparison of these two series of the curves shows that the effective medium theory reproduces the exact result within a wide range of wave vectors, $0<k d<0.5$. This is true not only for the propagating bands but also for the bandgap, where the wave vector is pure imaginary. This agreement is a strong evidence that the proposed homogenization scheme leads to the correct values of the effective parameters.

It follows from the graphs in Fig. 2 that the lower edge of the band gap $(\approx 96 \mathrm{kHz}$ ) coincides with the frequency where $\rho_{\text {eff }}(\omega)=0$ and the higher edge $(\approx 140 \mathrm{kHz})$ coincides with the frequency where $1 / \lambda_{\text {eff }}(\omega)=0$. The same tendency continues for the gaps lying at higher frequencies and it can be explained as follows. The frequencies of the bandgap edges at the $\Gamma$-point are the solutions of Eq. (1) at $k=0$. For high-contrast materials $\left(\omega b / c_{b} \ll 1\right)$ the righthand side of the dispersion Eq. (1) is reduced to

$$
\sin \left(\omega a / 2 c_{a}\right)\left[\rho_{a} a+\rho_{b} b\left(\omega a / 2 c_{a}\right) \cot \left(\omega a / 2 c_{a}\right)\right]=0 .
$$

The factor in square brackets vanishes exactly at the same frequencies when $\rho_{\text {eff }}(\omega)=0$. It follows from the band structure in Fig. 2 that equation $\rho_{\text {eff }}(\omega)=0$ defines a series of optical frequencies for the even bands. The optical frequencies for the odd bands are given by zeros of the factor $\sin \left(\omega a / 2 c_{a}\right)$ in Eq. (13). While these frequencies are not exactly the solutions of the equation $\lambda_{\text {eff }}^{-1}(\omega)=0$, but these two series of frequencies lie close to each other since $b / \lambda_{b} \ll a / \lambda_{a}$, at least for several low-lying optical frequencies. For the higher bands the factor $2 c_{a} / \omega a$ reduces the contribution of the term with $\sin \left(\omega a / 2 c_{a}\right)$ in Eq. (11), therefore the compliance $\lambda_{\text {eff }}^{-1}(\omega)$ does not vanish exactly at the optical frequencies of the odd bands.

\section{Conclusions}

In summary, it is proposed a new analytical approach for homogenization of acoustic superlattices which is valid in the long-wavelength limit but not necessarily low frequencies. The effective acoustic parameters of a homogeneous medium are introduced by equating the dynamics of the unit cell of the structure to the dynamics of the homogeneous layer of the same width . The calculated effective parameters - elastic modulus and mass density - exhibit strong frequency dependence. It is shown that the dispersion relation for longitudinal sound propagating parallel to the superlattice axis can be obtained from $\omega=k \sqrt{\lambda_{\text {eff }}(\omega) / \rho_{\text {eff }}(\omega)}$. For the long-wavelength sound this dispersion relation asymptotically coincides with the exact dispersion relation obtained from the Rytov equation. The proposed method can be equally applied to shear horizontal waves. It is shown that a layered structure may serve as a metamaterial with negative index of refraction. Unlike the known 2D acoustic metamaterials exhibiting negative refraction due to internal resonances, negative refraction in a layered 1D structure is due to anomalous dispersion. Demonstration of the effect of negative refraction will require calculation of the effective parameters for sound wave propagating at arbitrary angle with respect to the superlattice axis. Since a layered structure homogenizes to a uniaxial crystal, the mass density becomes a second-rank tensor. These calculations will be published elsewhere.

\section{Acknowledgments}

This work is supported by an Emerging Frontiers in Research and Innovation grant from the National Science Foundation (Grant No. 1741677).

1. M.S. Kushwaha, P. Halevi, L. Dobrzynski, and B. DjafariRouhani, Phys. Rev. Lett. 71, 2022 (1993).

2. M. Sigalas and E.N. Economou, Solid State Commun. 86, 141 (1993).

3. M.S. Kushwaha, Int. J. Mod. Phys. B 10, 977 (1996).

4. J.G. Barryman, J. Acoust. Soc. Am. 68, 1809 (1980).

5. J.G. Barryman, J. Acoust. Soc. Am. 68, 1820 (1980).

6. M. Kafesaki, R.S. Penciu, and E.N. Economou, Phys. Rev. Lett. 84, 6050 (2000).

7. A.A. Krokhin, J. Arriaga, and L.N. Gumen, Phys. Rev. Lett. 91, 264302 (2003).

8. Qing Ni and Jianchun Cheng, Phys. Rev. B 72, 014305 (2005).

9. Daniel Torrent, Andreas Håkansson, Francisco Cervera, and José Sánchez-Dehesa, Phys. Rev. Lett. 96, 204302 (2006).

10. Ying Wu, Yun Lai, and Zhao-Qing Zhang, Phys. Rev. B 76, 205313 (2007).

11. L. Gumen, J. Arriaga, and A. Krokhin, Fiz. Nizk. Temp. 37, 1221 (2011) [Low Temp. Phys. 37, 975 (2011)].

12. R.V. Craster, J. Kaplunov, and A.V. Pichugin, Proc. R. Soc. A 466, 2341 (2010).

13. A.N. Norris, A.L. Shuvalov, and A.A. Kutsenko, Proc. $R$. Soc. A 468, 1629 (2012). 
14. D. Torrent, Y. Pennec, and B. Djafari-Rouhani, Phys. Rev. B 92, 174110 (2015).

15. D. Torrent and José Sánchez-Dehesa, New J. Phys. 13, 093018 (2011).

16. Min Yang, Guancong Ma, Ying Wu, Zhiyu Yang, and Ping Sheng, Phys. Rev. B 89, 064309 (2014).

17. A. Konovalenko, E. Gutiérrez-Reyes, A.L. González, J. Flores-Méndez, and F. Pérez-Rodrguez, J. Appl. Phys. 121, 155102 (2017).

18. S.M. Rytov, Sov. Phys. Acoust. 2, 68 (1956).

19. S. Nemat-Nasser, J.R. Willis, A. Srivastava, and A.V. Amirkhizi, Phys. Rev. B 83, 104103 (2011).

20. I.S. Solís-Mora, M.A. Palomino-Ovando, and F. PérezRodríguez, J. Appl. Phys. 113, 093508 (2013).

21. Xiaoming Zhou and Gengkai Hu, Acta Mech. 224, 1233 (2013).

22. S.H. Lee, C.M. Park, Y.M. Seo, Z.G. Wang, and C.K. Kim, Phys. Lett. A 373, 4464 (2009); S.H. Lee, Ch.M. Park, Y.M. Seo, Zh.G. Wang, and Ch.K. Kim, Phys. Rev. Lett. 104, 054301 (2010).

23. S.A. Cummer, J. Christensen, and A. Alú, Nat. Rev. Mater. 1, 16001 (2016).

24. Mike Haberman and Matthew Guild, Phys. Today 69, 43 (2016).

25. V.G. Veselago, Sov. Phys. Usp. 10, 509 (1968).

26. L. Feng, X.-P. Liu, M.-H. Lu, Y.-B. Chen, Y.-F. Chen, Y.-W. Mao, J. Zi, Y.-Y. Zhu, S.-N. Zhu, and N.-B. Ming, Phys. Rev. B 73, 193101 (2006).

27. J. Bucay, E. Roussel, J.O. Vasseur, P.A. Deymier, A.-C. Hladky-Hennion, Y. Pennec, K. Muralidharan, B. DjafariRouhani, and B. Dubus, Phys. Rev. B 79, 214305 (2009).

28. Hélène Pichard, Olivier Richoux, and Jean-Philippe Groby, J. Acoust. Soc. Am. 132, 2816 (2012).

29. J. Florez-Méndez and F. Pérez-Rodríguez, Europhys. Lett. 103, 54001 (2013).

30. E. Gutiérrez-Reyes, J. Flores-Méndez, A.L. González, and F. Pérez-Rodríguez, Proc. SPIE (2015) pp. 94381H-94381H-10.

31. V. Cerdán-Ramírez, B. Zenteno-Mateo, M.P. Sampedro, M.A. Palomino-Ovando, B. Flores-Desirena, and F. PérezRodríguez, J. Appl. Phys. 106, 103520 (2009).
Динамічні ефективні параметри пружної надгратки з акустично різними компонентами

\section{Ю. Зубов, Bahram Djafari-Rouhani, A. Крохін}

Отримано аналітичні формули для частотно-залежних ефективного модуля пружності та ефективної щільності періодичної шаруватої структури. Запропонована процедура гомогенізації справедлива при досить високих частотах, що перевищують частоту першої забороненої зони в акустичному спектрі структури. Показано, що залежні від частоти ефективні параметри можуть набувати від'ємних значень або в різних частотних областях, або в одній досить вузькій області. Ця властивість показує, що одновимірна пружна структура в межі малих блоховських хвильових векторів може поводитися як акустичний метаматериал, у якого обидва ефективні параметри від’ємні.

Ключові слова: пружна надгратка, модулі пружності, періодична шарувата структура.

\section{Динамические эфффективные параметры упругой сверхрешетки с акустически различными компонентами}

\section{Ю. Зубов, Bahram Djafari-Rouhani, А. Крохин}

Получены аналитические формулы для частотнозависимых эффективного модуля упругости и эффективной плотности периодической слоистой структуры. Предложенная процедура гомогенизации справедлива при достаточно высоких частотах, превышающих частоту первой запрещенной зоны в акустическом спектре структуры. Показано, что зависящие от частоты эффективные параметры могут принимать отрицательные значения либо в различных частотных областях, либо в одной достаточно узкой области. Данное свойство показывает, что одномерная упругая структура в пределе малых блоховских волновых векторов может вести себя как акустический метаматериал, у которого оба эффективных параметра отрицательны.

Ключевые слова: упругая сверхрешетка, модули упругости, периодическая слоистая структура. 